\title{
Estudo dos efeitos de ambiente sobre as características lineares de tipo em rebanhos bovinos da raça Holandesa
}

[Study of environmental effects on linear type traits in Brazilian Holstein]

\author{
A.M.C. Esteves ${ }^{1}$, J.A.G. Bergmann ${ }^{1}$, M.C. Durães ${ }^{2}$, C.N. Costa ${ }^{2}$, H.M. Silva ${ }^{1}$ \\ ${ }^{1}$ Escola de Veterinária da UFMG \\ Caixa Postal 567 \\ 30123-970 - Belo Horizonte, MG \\ ${ }^{2}$ EMBRAPA - Gado de Leite - Juiz de Fora, MG
}

\begin{abstract}
RESUMO
Efeitos de estádio da lactação, idade à classificação e grupo contemporâneo de rebanho, ano e estação de classificação foram considerados nas análises de 21 características lineares de tipo e pontuação final de 2.122 animais da raça Holandesa, filhos de 178 reprodutores e nascidos em 83 rebanhos. Utilizou-se o método dos quadrados mínimos nas análises de variância das características. O efeito de estádio da lactação teve influência sobre as características do sistema mamário, evidenciando que houve alterações morfológicas durante o período de lactação para todas as características de úbere, exceto inserção do úbere anterior, textura do úbere e comprimento dos tetos. Dentre as características de conformação/capacidade corporal, profundidade corporal foi a mais influenciada pelo estádio da lactação, seguida do tamanho corporal, largura torácica e força lombar. Para todas as características de conformação/capacidade corporal o efeito da idade à classificação revelou-se altamente significativo. Dentre as características lineares da categoria pernas/pés, a variável idade à classificação foi importante $(\mathrm{P}<0,05)$ apenas para vista lateral das pernas. $\mathrm{O}$ efeito de idade sobre as características de garupa variou consideravelmente, sendo importante apenas para largura $(\mathrm{P}<0,01)$. Pontuação final e angulosidade também foram influenciadas $(\mathrm{P}<0,01)$ pelo efeito da idade. A verificação de significância estatística indicou a existência de componentes de meio e evidenciou a importância do estudo desses componentes.
\end{abstract}

Palavras-chave: bovino, característica linear de tipo, efeito de ambiente, Holandês

\begin{abstract}
Stage of lactation, classification age and herd-year-season contemporary group effects were considered in the analyses of 21 linear type traits, in addition to final score using 2,122 Brazilian Holstein cows, from 83 herds and daughters of 178 sires. Least square methodology was applied in the analyses of variance. With the exception of fore udder attachment, udder texture and fore teat length, the effect of stage of lactation was important $((\mathrm{P}<.05)$ for all other mammary system traits, indicating that there were morphological changes during lactation. All traits related to conformation/body capacity were influenced by classification age. Among these traits, body deep was the one affected by stage of lactation, followed by body size, chest width and loin strength. Among traits related to feet and legs, the effect of age of the animal on side view of rear legs was important $(\mathrm{P}<.05)$. The effect of age on traits related to rump was variable, being important $(\mathrm{P}<.05)$ only for rump width. Final score and angularity were also influenced by age $(\mathrm{P}<.05)$. This study revealed the importance of environment type trait effects on linear type trait evaluations.
\end{abstract}

Keywords: cattle, linear type trait, environment effect, Holstein

Recebido para publicação em 16 de outubro de 2003

Recebido para publicação, após modificações, em 23 de março de 2004

E-mail: alessandro@genespecuaria.com.br 


\section{INTRODUÇÃO}

Entende-se como classificação linear a metodologia aplicada para avaliação das medidas de conformação dos animais utilizada pelas associações de criadores de gado leiteiro.

Para aumentar a produção leiteira por meio da seleção de animais funcionais e produtivos é necessário identificar e quantificar os fatores de meio ambiente que influenciam as características consideradas na classificação linear para tipo. Estudos mostram que há influência dos fatores de meio como rebanho, ano e estação de classificação, estádio da lactação, idade ou ordem do parto, classificador, dentre outros, sobre as características lineares de tipo (Wilcox et al., 1959; Norman e Vanvleck, 1972a; Rennie et al., 1974; Thompson et al., 1983; Lawstuen et al., 1987).

Lucas et al. (1984) e Funk et al. (1991) estudaram os efeitos de meio sobre as características de conformação em vacas da raça Holandesa, encontrando efeitos significativos da idade da vaca, do estádio da lactação e do classificador sobre algumas características de tipo. Os efeitos de mês da parição, idade ao parto e estádio da lactação foram observados nos trabalhos de Cue et al. (1996). Lawstuen et al. (1987) trabalharam com grupos de idades dos animais à classificação e encontraram efeito significativo para os diferentes grupos

Muitos trabalhos enfocam a maneira como os efeitos de ambiente deveriam ser considerados nos estudos das características lineares. Alguns autores incluíram esses efeitos diretamente nos modelos estatísticos para ajustes simultâneos (Schaeffer, 1983; Thompson et al., 1983; Lawstuen et al., 1987). Outros calcularam constantes de ajustamentos para os diversos fatores de ambiente (Cassell et al., 1973; Norman et al., 1978; Norman et al., 1983; Foster et al., 1988).

Este trabalho teve por objetivo estudar os efeitos do estádio da lactação, idade à classificação e grupo contemporâneo rebanho-ano-estação de classificação sobre as características lineares de tipo de animais da raça Holandesa criados em Minas Gerais.

\section{MATERIAL E MÉTODOS}

Os dados de classificação linear foram coletados por técnicos da Associação de Criadores de Gado Holandês de Minas Gerais (ACGHMG), de 1995 a 1998. O arquivo de classificação linear, com 2.122 observações, incluiu 21 características lineares, com resultados de escores que variaram de 1 (um) a 9 (nove) pontos, mais a pontuação final, que variou de 55 a 90 pontos. As vacas foram classificadas uma vez em cada lactação. Ao todo foram avaliados animais de 83 rebanhos, progênies de 178 touros.

O sistema de classificação linear atualmente adotado pela raça Holandesa no Brasil é uma adaptação do sistema canadense. Nele, as características lineares são divididas em cinco seções. A distribuição das pontuações varia de acordo com as mensurações ou avaliações visuais observadas pelos classificadores para cada característica linear (Tab. 1).

A consideração das diferentes idades quando da avaliação e classificação dos animais foi feita incorporando-se esse fator nos modelos de análise como efeito fixo categórico, de acordo com os seguintes quatro grupos de idade: vacas com idade inferior a 1051 dias, vacas com idade entre 1051 e 1410 dias, vacas com idade entre 1411 e 1770 dias e vacas com idade superior a 1770 dias. Foram eliminados registros de animais com idade inferior a 610 dias à época da classificação linear.

A partir das informações geradas pelas datas do parto e datas da classificação foram obtidas 10 classes de estádio da lactação à época das avaliações lineares, a partir da classe com menos de 31 dias de lactação até a classe com mais do que 270 dias de lactação. Restrições também foram aplicadas e resultaram na eliminação de dados de animais classificados com menos de três dias e acima de 360 dias de lactação. 
Tabela 1. Escores aplicados para as características lineares de tipo, dentro das diversas seções, de acordo com mensurações diretas ou avaliações visuais

\begin{tabular}{lccc}
\hline Seções e características lineares & 1 ponto & 5 pontos & 9 pontos \\
\hline Conformação/Capacidade & & & \\
Estatura & $\leq 1,27 \mathrm{~m}$ & Nivelada & $\geq 1,47 \mathrm{~m}$ \\
Nivelamento da linha superior & Baixa & $=520 \mathrm{~kg}$ & Alta \\
Tamanho & $\leq 430 \mathrm{~kg}$ & Intermediária & $\geq 610 \mathrm{~kg}$ \\
Largura torácica & Estreita & Intermediária & Larga \\
Profundidade corporal & Rasa & Intermediária & Profunda \\
Força lombar & Fraca & & Forte \\
Garupa & & Nivelada (=5cm) & Baixa \\
Nivelamento da garupa & Alta & Média (=15cm) & Larga ( $\geq 20 \mathrm{~cm})$ \\
Largura da garupa & Estreita $(\leq 8 \mathrm{~cm})$ & & \\
Pernas/Pés & & Intermediário & Escarpado \\
Angulo do casco & Achinelado & Intermediária & Plano e limpo \\
Qualidade óssea & Grosseiro & Intermediária & Curvas \\
Vista lateral das pernas e pés & Retas & & \\
Sistema mamário & & Intermediária & Forte \\
Inserção do úbere anterior & Fraca & Centralizadas & Juntas \\
Colocação das tetas anteriores & Afastadas & Intermediárias & Longas \\
Comprimento das tetas & Curtas & Média (=27cm) & Alto ( $\geq 36 \mathrm{~cm})$ \\
Altura do úbere posterior & Baixo & Média (=15cm) & Largo ( $\geq 20 \mathrm{~cm})$ \\
Largura do úbere posterior & Estreito $(\leq 8 \mathrm{~cm})$ & Centralizadas & Juntas \\
Colocação das tetas posteriores & Afastadas & Intermediário & Raso \\
Profundidade do úbere & Profundo & Intermediário & Macio \\
Textura do úbere & Carnudo & Intermediário & Bem definido \\
Ligamento suspensório mediano & Pouco definido & & \\
Caracterização leiteira & & Intermediária & Angulosa \\
Angulosidade & Grosseira & &
\end{tabular}

A diversidade dos rebanhos e anos em que ocorreram as classificações, bem como diferenças segundo a estação climática do ano, foram considerados na modelagem dos escores lineares. Os meses de classificação foram agrupados em duas estações. A primeira considerou as classificações que ocorreram de abril a setembro de cada ano e a segunda as classificações de outubro do mesmo ano a março do ano subseqüente. Assim, os efeitos de rebanho, ano e estação de classificação foram combinados em um único efeito fixo rebanho-ano-estação de classificação (RAEC). As classes RAEC foram formadas com o objetivo de reduzir o número de efeitos fixos no modelo e, conseqüentemente, as necessidades computacionais.

Além da variável RAEC, o modelo utilizado para as características lineares também incluiu os efeitos fixos de estádio da lactação, idade do animal e o efeito aleatório do pai da vaca. Como os dados obtidos da ACGHMG não apresentavam referências específicas aos classificadores, esse efeito não foi considerado nas análises.

O modelo matemático adotado foi

$\mathrm{Y}_{\mathrm{ijklm}}=\mu+\mathrm{RAEC}_{\mathrm{i}}+\mathrm{A}_{\mathrm{j}}+\mathrm{L}_{\mathrm{k}}+\mathrm{S}_{1}+\mathrm{e}_{\mathrm{ijklm}}$, em que:

$\mathrm{Y}_{\mathrm{ijklm}}=$ observação para cada característica linear do indivíduo $\mathrm{m}$;

$\mu=$ média da população;

$\mathrm{RAEC}_{\mathrm{i}}=$ efeito fixo da subclasse i para rebanho, ano e estação de classificação;

$A_{j}=$ efeito fixo da subclasse $\mathrm{j}$ para idade da vaca à classificação;

$\mathrm{L}_{\mathrm{k}}=$ efeito fixo da subclasse $\mathrm{k}$ para estádio da lactação;

$\mathrm{S}_{1}=$ efeito aleatório do pai da vaca;

$\mathrm{e}_{\mathrm{m}}=$ efeito aleatório do erro associado à cada observação. 
O método utilizado nas análises foi o dos quadrados mínimos, por meio de análises de variância, utilizando o procedimento PROC GLM do pacote estatístico SAS (User's..., 1995).

\section{RESULTADOS E DISCUSSÃO}

O efeito de estádio da lactação teve influência $(\mathrm{P}<0,01)$ sobre as características do sistema mamário, evidenciando que houve alterações morfológicas durante o período de lactação para todas as características de úbere, exceto inserção do úbere anterior, textura do úbere $\mathrm{e}$ comprimento dos tetos (Tab. 2 e 3). O estádio de lactação respondeu por $44 \%$ das causas de variação para a característica colocação de tetos posteriores, 35\% para colocação de tetos anteriores, $31 \%$ para altura do úbere e $39 \%$ para ligamento mediano. Mcmanus e Saueressig (1997), pesquisando rebanho Holandês nacional, também observaram efeito do estádio da lactação sobre o sistema mamário, sendo a largura do úbere posterior a característica mais influenciada.

Tabela 2. Resumo das análises de variâncias das características lineares do sistema mamário (úbere anterior e úbere posterior)

\begin{tabular}{|c|c|c|c|c|c|c|c|c|c|c|c|c|}
\hline \multirow[t]{2}{*}{ Fonte de variação } & \multicolumn{2}{|c|}{$\begin{array}{l}\text { Inserção } \\
\text { do úbere }\end{array}$} & \multicolumn{2}{|c|}{$\begin{array}{c}\text { Comprimento } \\
\text { das tetas }\end{array}$} & \multicolumn{2}{|c|}{$\begin{array}{l}\text { Colocação das } \\
\text { tetas anteriores }\end{array}$} & \multicolumn{2}{|c|}{$\begin{array}{c}\text { Colocação das } \\
\text { tetas posteriores }\end{array}$} & \multicolumn{2}{|c|}{$\begin{array}{c}\text { Altura } \\
\text { do úbere }\end{array}$} & \multicolumn{2}{|c|}{$\begin{array}{l}\text { Largura } \\
\text { do úbere }\end{array}$} \\
\hline & Gl & QM & Gl & QM & Gl & QM & Gl & QM & Gl & QM & Gl & QM \\
\hline RAEC & 73 & $5,78^{* *}$ & 82 & $2,34 * *$ & 73 & $3,00 * *$ & 73 & $2,73 * *$ & 61 & $2,54 * *$ & 73 & $8,55 * *$ \\
\hline Estádio da lactação & 9 & $2,01 \mathrm{~ns}$ & 9 & $1,39 \mathrm{~ns}$ & 9 & $2,31 *$ & 9 & $4,21 * *$ & 9 & $3,34 * *$ & 9 & $6,74 * *$ \\
\hline Idade do animal & 3 & $1,22 \mathrm{~ns}$ & 3 & $15,33 * *$ & 3 & $0,46 \mathrm{~ns}$ & 3 & $1,48 \mathrm{~ns}$ & 3 & $4,00 * *$ & 3 & $56,83 * *$ \\
\hline Total & 1539 & 10,24 & 1937 & 19,93 & 1539 & 6,58 & 1539 & 9,54 & 1539 & 10,72 & 1539 & 73,51 \\
\hline
\end{tabular}

Tabela 3. Resumo das análises de variâncias das características lineares do sistema mamário (úbere) e pontuação final

\begin{tabular}{lcccccccc}
\multirow{2}{*}{ Fonte de variação } & \multicolumn{2}{c}{ Profundidade do úbere } & \multicolumn{2}{c}{ Ligamento mediano } & \multicolumn{2}{c}{ Textura do úbere } & \multicolumn{2}{c}{ Pontuação final } \\
\cline { 2 - 9 } & Gl & QM & Gl & QM & Gl & QM & Gl & QM \\
\hline RAEC & 73 & $5,03^{* *}$ & 73 & $6,75^{* *}$ & 82 & $4,68^{* *}$ & 82 & $48,82^{* *}$ \\
Estádio da lactação & 9 & $3,58^{* *}$ & 9 & $5,45^{* *}$ & 9 & $2,45 \mathrm{~ns}$ & 9 & $10,44 \mathrm{~ns}$ \\
Idade do animal & 3 & $36,33^{* *}$ & 3 & $0,29 \mathrm{~ns}$ & 3 & $4,29^{*}$ & 3 & $696,00^{* *}$ \\
Total & 1539 & 45,90 & 1539 & 13,90 & 1937 & 12,82 & 1937 & 762,60 \\
\hline
\end{tabular}

**(P<0,01); *(P<0,05); ns (não significativo)

$\mathrm{RAEC}=$ grupo contemporâneo de rebanho, ano e estação de classificação.

Nem todas as características de conformação/capacidade corporal foram influenciadas pelo estádio de lactação (Tab. 4). Profundidade corporal foi a mais influenciada, seguida do tamanho corporal, largura torácica e força lombar. O tamanho corporal reflete o peso do animal, desse modo há a tendência de os animais pontuarem mais alto a partir do meio para o fím da lactação. Estes resultados são explicados pelo fato de as vacas apresentarem balanço energético negativo no início da lactação e por coincidirem com o pico de lactação. A partir do meio da lactação ocorre o pico de ingestão de alimentos e a diminuição da produção, com conseqüente acúmulo de reservas corporais e aumento do peso. A possível gestação da vaca também contribui para o aumento do peso corporal. Hietanen e Ojala (1995) relataram que vacas classificadas para tamanho, do segundo ao quarto mês da lactação, apresentaram-se mais leves à avaliação do peso corporal. White (1974) verificou que o estádio da lactação influencia as características de conformação/capacidade corporal, mas seu efeito é pequeno. 
Tabela 4. Resumo das análises de variâncias das características lineares de conformação/capacidade

\begin{tabular}{|c|c|c|c|c|c|c|c|c|c|c|c|c|}
\hline \multirow[t]{2}{*}{ Fonte de variação } & \multicolumn{2}{|c|}{ Estatura } & \multicolumn{2}{|c|}{$\begin{array}{l}\text { Nivelamento da } \\
\text { linha superior }\end{array}$} & \multicolumn{2}{|c|}{ Tamanho } & \multicolumn{2}{|c|}{ Largura torácica } & \multicolumn{2}{|c|}{$\begin{array}{c}\text { Profundidade } \\
\text { corporal }\end{array}$} & \multicolumn{2}{|c|}{ Força lombar } \\
\hline & Gl & QM & Gl & QM & Gl & QM & Gl & QM & Gl & QM & Gl & QM \\
\hline$\overline{\text { RAEC }}$ & 73 & $8,47 * *$ & 82 & $1,95 * *$ & 73 & $12,80 * *$ & 82 & $7,44 * *$ & 97 & $2,65 * *$ & 82 & $4,25 * *$ \\
\hline Estádio da lactação & 9 & $0,91 \mathrm{~ns}$ & 9 & $0,72 \mathrm{~ns}$ & 9 & $3,16^{*}$ & 9 & $2,50 *$ & 9 & $2,26 * *$ & 9 & $2,65^{*}$ \\
\hline Idade do animal & 3 & $6,61 * *$ & 3 & $54,05 * *$ & 3 & $28,48 * *$ & 3 & $68,12 * *$ & 3 & $22,26 * *$ & 3 & $27,06 * *$ \\
\hline Total & 1539 & 17,74 & 1637 & 57,34 & 1539 & 45,91 & 1935 & 79,15 & 1538 & 29,03 & 1936 & 35,23 \\
\hline
\end{tabular}

$* *(\mathrm{P}<0,01) ; *(\mathrm{P}<0,05) ;$ ns (não significativo).

RAEC = grupo contemporâneo de rebanho, ano e estação de classificação.

Hansen et al. (1969) demonstraram ser o efeito do estádio da lactação significativo para todas as características lineares de conformação/capacidade corporal, sistema mamário, caracterização leiteira, aparência geral e pontuação final. Evidenciaram que as características têm tendência de se apresentarem com maior pontuação no início e no final da lactação, semelhante aos resultados de Wilcox et al. (1959).

Ausência de significância estatística do efeito de estádio da lactação foi observada para as características de garupa e de pernas/pés. Estes resultados foram semelhantes aos de Norman et al. (1978) e Thompson et al. (1983) que concluíram não ser importante o efeito de estádio de lactação para pernas/pés.

Muitos autores concluem ser o efeito da idade à classificação um dos mais importantes na avaliação das características lineares (Wilcox et al., 1959; Hansen et al., 1969; White, 1974; Norman et al., 1983; Cue et al., 1996). Para White (1974), de todos os fatores de ambiente que podem influenciar os resultados de escores lineares de vacas leiteiras, a idade do animal à classificação é o mais importante. Bowden, (1982) comenta que são esperadas alterações para as características de tipo com o avançar da idade, algumas melhoram e outras pioram.

Para todas as características de conformação/capacidade corporal o efeito da idade à classificação revelou-se altamente significativo. Este efeito poderia ser facilmente explicado pelo fato de os animais nas primeiras lactações ainda não terem atingido a maturidade fisiológica. Sabe-se que animais mais velhos são mais profundos, mais pesados, mais altos em sua porção anterior (ascendentes), com peito mais largo e lombo mais forte.

Dentre as características lineares da categoria pernas/pés, a variável idade à classificação foi importante $(\mathrm{p}<0,05)$ apenas para vista lateral das pernas (Tab. 5). Ausência do efeito da idade sobre as características de pernas/pés também foi verificada por Wilcox et al. (1959), Hansen et al. (1969) e Rennie et al. (1974). Ainda, os efeitos de idade sobre as características de garupa variaram consideravelmente, sendo importantes apenas para largura $(\mathrm{P}<0,01 ;$ Tab. 5).

Tabela 5. Resumo das análises de variâncias das características lineares de garupa, pernas/pés e caracterização leiteira

\begin{tabular}{|c|c|c|c|c|c|c|c|c|c|c|c|c|}
\hline \multirow[t]{2}{*}{ Fonte de variação } & \multicolumn{2}{|c|}{$\begin{array}{c}\text { Nivelamento } \\
\text { da garupa }\end{array}$} & \multicolumn{2}{|c|}{$\begin{array}{c}\text { Largura } \\
\text { da garupa }\end{array}$} & \multicolumn{2}{|c|}{$\begin{array}{l}\text { Ângulo } \\
\text { do casco }\end{array}$} & \multicolumn{2}{|c|}{$\begin{array}{l}\text { Qualidade } \\
\text { óssea }\end{array}$} & \multicolumn{2}{|c|}{$\begin{array}{c}\text { Vista lateral } \\
\text { das pernas }\end{array}$} & \multicolumn{2}{|c|}{ Angulosidade } \\
\hline & Gl & $\mathrm{QM}$ & Gl & QM & Gl & QM & $\mathrm{Gl}$ & QM & Gl & QM & Gl & $\mathrm{QM}$ \\
\hline$\overline{\mathrm{RAEC}}$ & 82 & $1,45 * *$ & 73 & $7,58 * *$ & 73 & $5,05^{* *}$ & 82 & $4,86 * *$ & 73 & $3,37 * *$ & 73 & $5,97 * *$ \\
\hline Estádio da lactação & 9 & $0,55 \mathrm{~ns}$ & 9 & $1,25 \mathrm{~ns}$ & 9 & $1,28 \mathrm{~ns}$ & 9 & $1,42 \mathrm{~ns}$ & 9 & $2,25^{*}$ & 9 & $1,45 \mathrm{~ns}$ \\
\hline Idade do animal & 3 & $0,87 \mathrm{~ns}$ & 3 & $31,54 * *$ & 3 & $1,90 \mathrm{~ns}$ & 3 & $0,21 \mathrm{~ns}$ & 3 & $5,66 * *$ & 3 & $36,13 * *$ \\
\hline Total & 1937 & 3,74 & 1539 & 42,08 & 1539 & 9,39 & 1937 & 7,97 & 1539 & 12,39 & 1539 & 44,68 \\
\hline
\end{tabular}


A pontuação final e a angulosidade também foram altamente influenciadas $(\mathrm{P}<0,01)$ pelo efeito de idade (Tab. 3 e 5). Consideração importante a respeito do efeito dessa variável sobre a pontuação final é que os animais de primeira lactação recebem, no máximo, 88 pontos na classificação final. Outra implicação decorre da recomendação feita pela Associação da raça aos classificadores de atribuir mais de 90 pontos apenas para animais de terceira ordem de lactação ou acima.

Segundo Lucas et al. (1984), a idade foi importante fonte de variação para o escore linear das características estatura, força corporal, caracterização leiteira, largura da garupa, inserção do úbere anterior, largura do úbere posterior, profundidade do úbere e ligamentos. Para os autores, todas as características de conformação/capacidade são influenciadas pela idade à classificação. A ausência de significância estatística para o efeito de idade sobre as características vista lateral das pernas e colocação dos tetos, verificadas no presente estudo, estão de acordo com os resultados de Thompson et al. (1981). Entretanto, Thompson et al. (1983) concluíram que a idade do animal teve efeito significativo sobre todas características, exceto vista posterior das pernas e ângulo do casco.

$\mathrm{O}$ efeito dos grupos de RAEC foi importante $(\mathrm{P}<0,01)$ causa de variação para todas as características lineares (Tab. 2, 3, 4 e 5). Este resultado era esperado e é um reflexo, principalmente, das diferenças de manejo, de alimentação, dos aspectos sanitários e das condições climáticas às quais os animais são submetidos. Para algumas características, o efeito de grupo contemporâneo RAEC revelou-se como a mais importante fonte de variação, respondendo por $61 \%$ da variação total para qualidade óssea, 53\% para ângulo de casco, 48\% para estatura e $38 \%$ para nivelamento de garupa. Os grupos de contemporâneos, considerando rebanho e ano de nascimento, foram importantes causas de variação das características lineares. Ainda, segundo Norman et al. (1978) e Norman et al. (1983), quando considerados em conjunto, esses efeitos responderam mais pela variação do que seus efeitos individuais de rebanho e de ano. Analisando separadamente os efeitos de rebanho e de ano sobre as características de tipo, Norman e Van Vleck (1972b) concluíram que o componente de variância atribuído ao ano de classificação foi de pequena magnitude para todas as características de tipo. Norman et al. (1978) concluíram que o efeito de rebanho foi mais importante do que o efeito de ano.

É importante considerar que no presente estudo pode ter havido certo confundimento entre os efeitos de rebanho e de classificador, uma vez que o segundo efeito não pôde ser avaliado. Além disso, pelas informações fornecidas pela Associação da raça, os técnicos geralmente classificam os mesmos rebanhos, em razão de sua divisão de atuação por área geográfica. Assim, pode-se supor que parte da importância dada ao efeito de RAEC sobre as características lineares decorre do efeito classificador. Esse mesmo confundimento foi relatado em outros trabalhos (Bowden, 1982; Norman et al., 1983).

O efeito isolado da estação de classificação sobre diversas características sempre foi considerado em pesquisas conduzidas em rebanhos de Minas Gerais, uma vez que o Estado tem dois períodos distintos, caracterizados por abundância e escassez de chuvas. Isto reflete no manejo nutricional das fazendas, avaliadas em função da alimentação oferecida em cada período.

\section{CONCLUSÕES}

A importância dos diversos efeitos de ambiente sobre as características lineares de tipo indica a necessidade de ajustamentos ou da formação de grupos de contemporâneos quando for feita a seleção e quando forem estimados parâmetros genéticos para essas características. Os resultados poderão servir de referência para a implementação de avaliações de touros e de vacas para as características lineares de tipo das raças leiteiras no Brasil.

\section{REFERÊNCIAS BIBLIOGRÁFICAS}

BOWDEN, V. Type classification in dairy cattle: a review. Anim. Breed. Abstr., v.50, p.147-161, 1982.

CASSEL, B.G.; VINSON, W.E.; WHITE, J.M. et al. Age correction factors for type traits in Holstein cows. J. Dairy Sci., v.56, p.1178, 1973. 
CUE，R.I.; HARRIS， B.L.; RENDEL，J.M. Genetics parameters for traits other than production in purebred and crossbred New Zealand dairy cattle. Livest. Prod. Sci., v.45, p.123-135, 1996.

FOSTER, W.W.; FREEMAN, A.E.; BERGER, P.J. et al. Linear type traits analysis with genetic parameter estimation. J. Dairy Sci., v.71, p.223$231,1988$.

FUNK, D.C.; HANSEN, L.B.; FUNK, D.A. Adjustment of linear type scores from Holstein classification for age and stage of lactation. $J$. Dairy Sci., v.74, p.645-650, 1991.

HANSEN, L.R.; BARR, G.R.; WIECKERT, D.A. Effects of age and stage of lactation on type classification. J. Dairy Sci., v.52, p.646-650, 1969.

HENDERSON, C.R. Estimation of variance and covariance components. Biometrics, v.9, p.226252, 1953.

HIETANEN, H.; OJALA, M. Factors affecting body weight and its association with milk production traits in Finnish Ayrshire and Friesian cows. Acta Agr. Scand., v.45, p.17-25, 1995.

LAWSTUEN, D.A.; HANSEN, L.B.; JOHNSON, L.P. Inheritance and relationships of linear type traits for age groups of Holsteins. $J$. Dairy Sci., v.70, p.1027-1035, 1987.

LUCAS, J.L.; PEARSON, R.E.; VINSON, W.E. et al. Experimental linear description type classification. J. Dairy Sci., v.67, p.1767-1775, 1984.

MCMANUS, C.; SAUERESSIG, M.G. Estudo das características lineares de tipo em gado Holandês em confinamento total no DF: análise de variância. In: REUNIÃO ANUAL DA SOCIEDADE BRASILEIRA DE ZOOTECNIA, 34., 1997, Juiz de Fora. Anais... Juiz de Fora: SBZ, 1997. p.71-73.

NORMAN, H.D.; CASSEL, B.G.; DAWDY,
M.L. Genetic and environmental effects influencing Guernsey type classification scores. J. Dairy Sci., v.66, p.127, 1983.

NORMAN, H.D.; CASSEL, B.G.; WRIGHT, E.E. Effect of herd, year, age, and stage of lactation on Jersey type classification. J. Dairy Sci., v.61, p.352, 1978.

NORMAN, H.D.; VAN VLECK, L.D. Type appraisal: I. Effects of age and stage of lactation on type ratings. J. Dairy Sci., v.55, p.1706-1716, 1972a.

NORMAN, H.D.; VAN VLECK, L.D. Type appraisal: II. Variation in type traits due to sires, herds and years. J. Dairy Sci., v.55, p.1717-1725, $1972 b$.

RENNIE, J.C.; BATRA, T.R.; FREEMAN, M.G. et al. Environmental and genetic parameters for type traits in Holstein cows. J. Dairy Sci., v.57, p.1221-1224, 1974.

SCHAEFFER, L.R. Estimates of variance and covariance components for Holstein type traits. Can. J. Anim. Sci., v.63, p.763. 1983.

THOMPSON, J.R.; FREEMAN, A.E.; WILSON, D.J. et al. Evaluation of a linear type program in Holsteins. J. Dairy Sci., v.64, p.16101617, 1981.

THOMPSON, J.R.; LEE, K.L.; FREEMAN, A.E. et al. Evaluation of linearized type appraisal system for Holstein cattle. J. Dairy Sci., v.66, p.325-331, 1983.

USER's guide: basic and statistics. Cary, NC: SAS Institute, 1995. 1290p.

WHITE, J.M. Genetic parameters of conformational and management traits. J. Dairy Sci., v.57, p.1267-1278, 1974.

WILCOX, C.J.; MATHER, R.E.; PFAU, K.O. et al. Changes in type ratings of Holstein cows due to age, season, stage of lactation, classifier and year. J. Dairy Sci., v.41, p.1065-1071, 1959. 